\title{
Criticality Calculations of the Very High Temperature Reactor Critical Assembly Benchmark with Serpent and SCALE/KENO-VI
}

Friederike Bostelmann ${ }^{a *}$, Hans R. Hammer ${ }^{b}$, Javier Ortensi ${ }^{c}$, Gerhard Strydom ${ }^{c}$, Kiril Velkov ${ }^{a}$, Winfried Zwermann ${ }^{a}$

a Gesellschaft für Anlagen- und Reaktorsicherheit (GRS) gGmbH, 85748 Garching, Germany.

${ }^{\mathrm{b}}$ Nuclear Engineering Department, Texas A\&M, College Station, TX 77840, USA.

${ }^{\mathrm{C}}$ Nuclear Science and Engineering Division, Idaho National Laboratory (INL), Idaho Falls, ID 83401, USA.

\begin{abstract}
Within the framework of the IAEA Coordinated Research Project on HTGR Uncertainty Analysis in Modeling, criticality calculations of the Very High Temperature Critical Assembly experiment were performed as the validation reference to the prismatic MHTGR-350 lattice calculations. Criticality measurements performed at several temperature points at this Japanese graphitemoderated facility were recently included in the International Handbook of Evaluated Reactor Physics Benchmark Experiments, and represent one of the few data sets available for the validation of HTGR lattice physics. This work compares VHTRC criticality simulations utilizing the Monte Carlo codes Serpent and SCALE/KENO-VI. Reasonable agreement was found between Serpent and KENO-VI, but only the use of the latest ENDF cross section library release, namely the ENDF/B-VII. 1 library, led to an improved match with the measured data. Furthermore, the fourth beta release of SCALE 6.2/KENO-VI showed significant improvements from the current SCALE 6.1.2 version, compared to the experimental values and Serpent.
\end{abstract}

Keywords: HTGR, VHTRC, Serpent, SCALE/KENO-VI

\section{Introduction}

In 2013, the IAEA launched the Coordinated Research Project (CRP) on Uncertainty Analysis in Modeling (UAM) to study uncertainty propagation in the High Temperature Gas-cooled Reactor (HTGR) analysis chain [1]. The CRP mirrors the current OECD/NEA UAM project to assess uncertainty propagation techniques for LWR, VVER and BWR designs [2]. HTGR reactors require lattice and core simulation techniques not typically utilized in Light Water Reactor (LWR) analysis due to several unique features, such as double heterogeneous fuel design including tristructural isotropic (TRISO) fuel particles, large graphite quantities, and high operational temperatures.

*Corresponding author: Friederike.Bostelmann@grs.de 
Within the framework of the CRP on HTGR UAM, two benchmark problems are defined, with the prismatic HTGR design represented by the 350 MW General Atomics Modular High

Temperature Gas-cooled Reactor (MHTGR) [3] and a 250 MW modular pebble bed design similar to the HTR-PM (INET, China) [1]. For each of these designs, several phases and subexercises were defined, starting with unit cell and lattice-equivalent calculations (Phase I) up to coupled system transients (Phase IV).

Exercise I-2a for the prismatic MHTGR-350 requires an infinite lattice calculation to be performed on a single fuel block. The objective is to address the uncertainties due to the basic nuclear data as well as the impact of processing the cross-section and covariance data, selection of multi-group structure, and double-heterogeneity or self-shielding treatment. Participants are then required to develop techniques for the propagation of these uncertainties to the next phase (full core simulation).

The Very High Temperature Reactor Critical Assembly (VHTRC) is a prismatic graphitemoderated single block assembly located at the Japan Atomic Energy Agency (JAEA) Tokai Research and Development Center in Japan. Data generated by VHTRC criticality measurements at several temperature points has been verified and included in the OECD/NEA International Handbook of Evaluated Reactor Physics Benchmark Experiments (IRPhEP) [4] as a validation benchmark experiment for graphite moderated temperature dependent multiplication factors. In addition to the uncertainty data specified for both the measured and calculated multiplication factors, uncertainties on manufacturing parameters are also provided.

Due to the limited availability of experimental uncertainty data, calculations of this assembly shall serve as reference to the MHTGR-350 single block calculations. As a first step, Monte Carlo criticality simulations of the VHTRC experiments performed with two Monte Carlo codes are compared. The Serpent code [5] offers the capability to model the double-heterogeneity of the fuel using a random particle distribution. The KENO-VI Monte Carlo Code of the SCALE code package [6] was also applied since, at a later stage, uncertainty calculations will be performed with the perturbation based SCALE/TSUNAMI module and with the sample-based approaches of SCALE/SAMPLER and the GRS tool XSUSA [7] which also utilizes SCALE modules. The results of the Monte Carlo simulations, including a comparison to the Japanese MVP-II simulations published in the IRPhEP, are described in this work.

\section{The VHTRC Experiment}

A detailed description of the VHTRC is provided in the IRPhEP Handbook [4], but a condensed version is included here for completeness. The VHTRC is a graphite-moderated thermal critical assembly that has a core loaded with pin-in-block fuel of low enriched uranium and a graphite reflector (Figure 1). The assembly has a hexagonal prism shape (2.4 $\mathrm{m}$ across the flats and 2.4 $\mathrm{m}$ long). In the radial and axial directions the assembly is covered with cadmium sheets as a thermal neutron absorber, steel frames, and alumina-silica fiber blankets as heat insulation. It can be heated up to $200^{\circ} \mathrm{C}$ using 40 electric heaters to study temperature effects on the assembly characteristics.

The assembly consists of two axially-jointed hexagonal-prism half assemblies of which one is fixed and the other is movable. The structures of the half assemblies are made of graphite 
blocks supported with steel frames. In general, unused holes of graphite blocks are filled with graphite rods.

The reflector blocks are classified into three types: (1) blocks having three large holes for control rods and three small holes for multi-purpose usage, (2) blocks having one small hole for multipurpose usage such as insertion of electric heaters, and (3) outermost half-blocks having one small hole.

The shape of the graphite blocks for the first two types are hexagonal, while the outermost blocks, located along the radial perimeter of the core, are trapezoidal to form a hexagonal assembly. The fuel block has 19 holes into which fuel rods, graphite rods, burnable poison rods, etc. can be inserted according to the experiment. Each fuel rod is paired with a solid graphite rod to reach an axial length of $1,200 \mathrm{~mm}$. The fuel rod is a stack of 20 annular fuel compacts packed in a cylindrical graphite sheath. The fuel compacts are made of coated fuel particles uniformly dispersed in a graphite matrix. The coated fuel particles are bi-structural isotropic (BISO) particles, which have two carbon layers surrounding a low-enriched uranium dioxide kernel. There are two types of fuel compacts (B-2 and B-4) containing 2 wt.\% and 4 wt.\% enriched uranium, respectively. On average, each fuel compact contains approximately 20,000 randomly distributed fuel particles.

The experiments were performed with three different loading patterns ( $\mathrm{HP}, \mathrm{HC}-1$, and $\mathrm{HC}-2)$ which differed in the number and type of fuel rods (see Figure 2 to Figure 4). In the HP core experiments, the assembly was first brought to a critical state at room temperature $\left(25.5^{\circ} \mathrm{C}\right)$. In the course of heating the whole core, the reactivity change was measured by the pulsed neutron method at four additional different temperatures $\left(71.2^{\circ} \mathrm{C}, 100.9^{\circ} \mathrm{C}, 150.5^{\circ} \mathrm{C}\right.$, and $\left.199.6^{\circ} \mathrm{C}\right)$. In the $\mathrm{HC}-1$ and $\mathrm{HC}-2$ core experiments criticality was attained at $8.0^{\circ} \mathrm{C}$ and $200.3^{\circ} \mathrm{C}$, respectively. In total, three critical and four subcritical configurations were measured. Due to reactivity effects of loading irregularities, the measured eigenvalues were, however, corrected with the result that the VHTRC dataset contains four critical and three subcritical core states. 


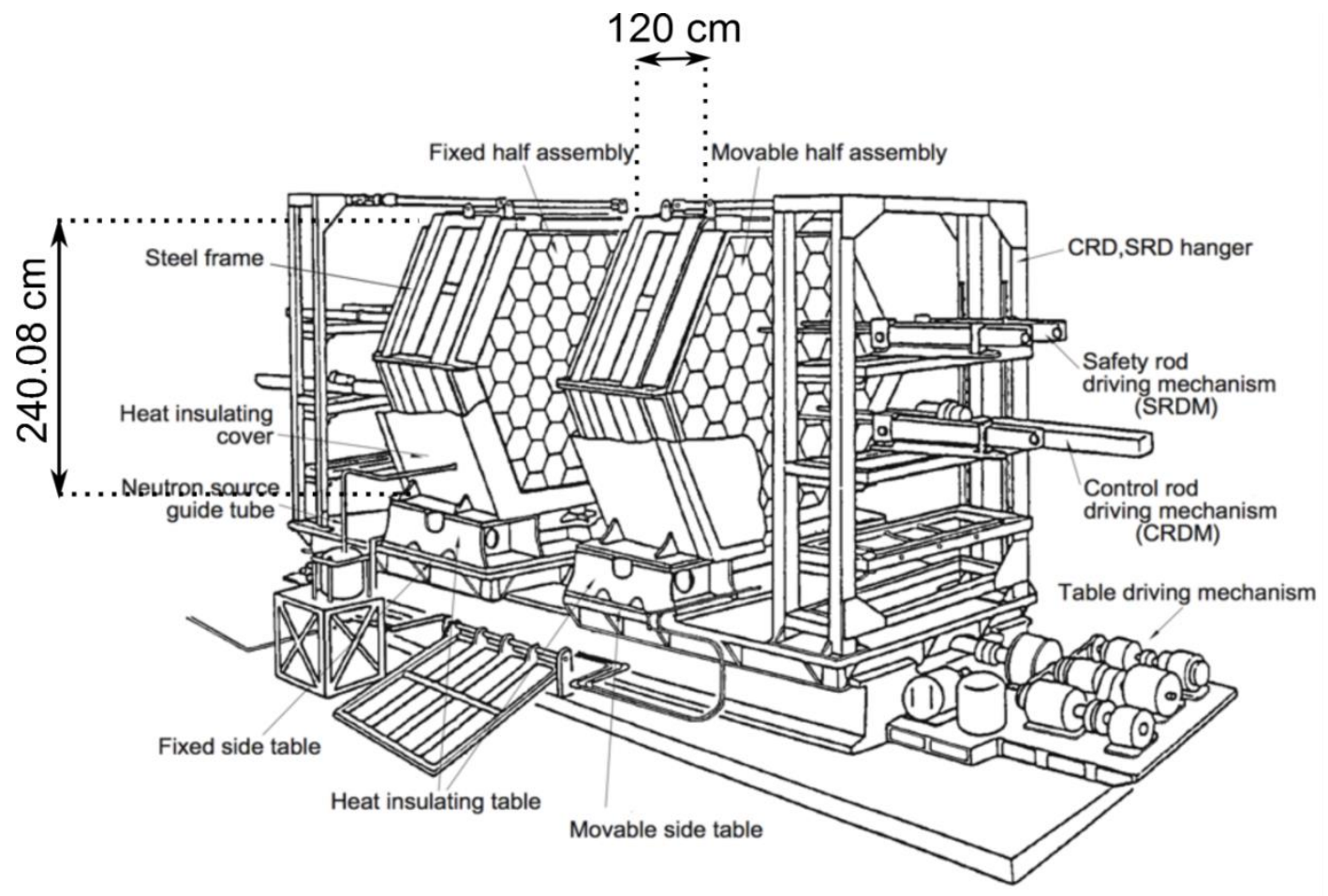

Figure 1: The VHTRC assembly [4].

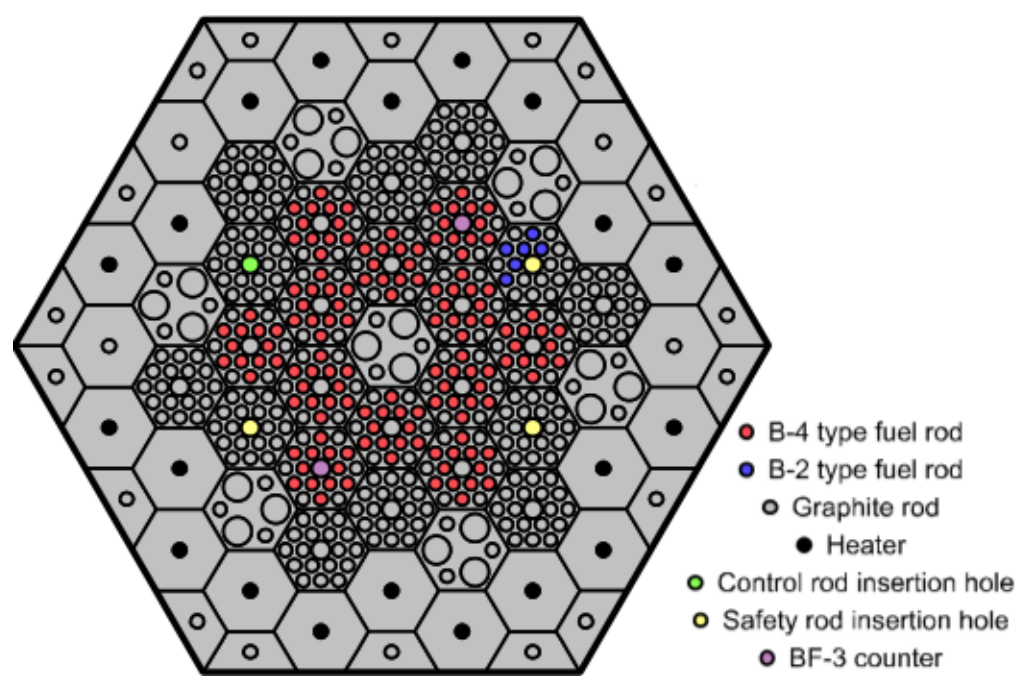

Figure 2: Core loading pattern of the HP core [4]. 


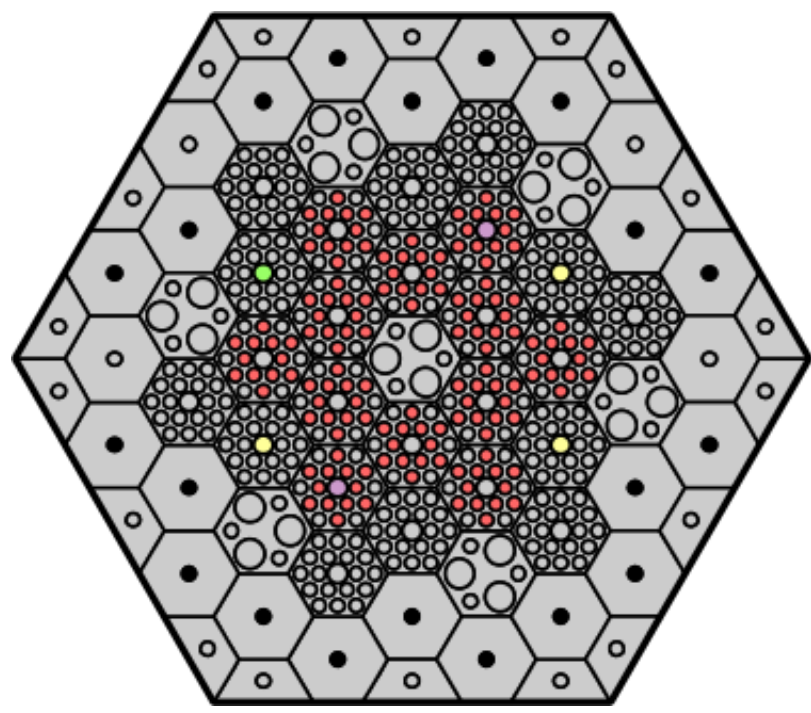

Figure 3: Core loading pattern of the $\mathrm{HC}-1$ core [4].

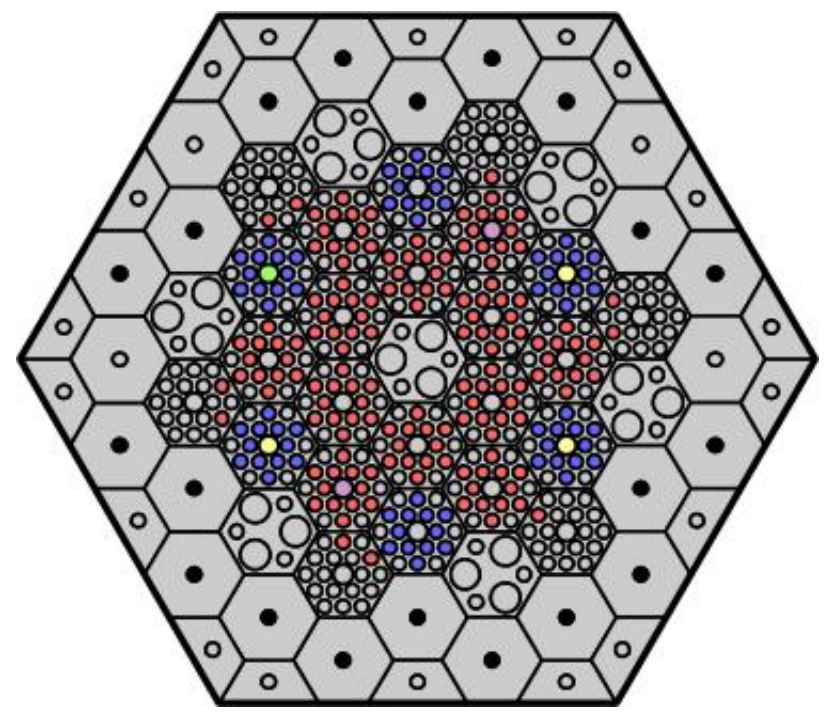

Figure 4: Core loading pattern of the HC-2 core [4].

\section{Computer Codes}

The calculations of the VHTRC were performed with the Monte Carlo codes Serpent 2 [5] and SCALE/KENO-VI [6].

Serpent is a 3-D continuous-energy neutron transport code, and has been developed at the VTT Technical Research Centre of Finland since 2004. It is capable of simulating various fuel assembly geometries, performing burn-up calculations, and can also be utilized for simulating smaller reactor cores. In order to provide a better parallelization of the program and the possibility of performing 3-D burn-up calculations, the Serpent code has been rewritten. For the calculations reported in this paper, a beta version of the so-called Serpent 2 (version 2.1.21) was used [8]. Since Serpent 2 provides the capability of performing parallel calculations using a combination of Message Passing Interface (MPI) and Open Multi-Processing (OMP) parallelization, the calculations were performed on the INL and GRS High Performance Computing systems to take advantage of these features.

The KENO-VI module of the SCALE 6.1 code package was developed at Oak Ridge National Laboratory (ORNL). It is a three-dimensional Monte Carlo criticality code that can read continuous-energy (CE) or multi-group (MG) ENDF/B-VII.0 cross section data.

In addition to the current SCALE 6.1.2 release, results generated by a beta release of SCALE 6.2 (version 4, referred to here as 6.2b4) have also been included in this study [9]. The beta version includes a new 252-group library that provides, among others, a more detailed representation of the ${ }^{238} \mathrm{U}$ resonance structure, improved resonance self-shielding parameters and an increase in the thermal scattering cut off. The relative performance of this library for this HTGR application is also reported here. In addition to the ENDF/B-VII.0 cross-section library, this beta version also implements the ENDF/B-VII.1 library, thus it enables calculations with the latest release. 


\section{Models}

\subsection{Serpent model with a random particle distribution}

The model utilized for this study is based on the final IRPhEP benchmark specifications. The fuel rods are composed of fuel compacts which are surrounded by graphite sheaths. These fuel rods are arranged in fuel blocks, the fuel blocks arranged in a lattice, and two of these lattices combined to form the reactor core. Graphite rods and three different graphite blocks are included as well. It should be noted that additional features that were present in the experiment (cadmium sheets and steel frames around the assemblies) were not included in the final IRPhEP benchmark specifications, and are therefore also excluded from the models utilized in this study.

To include randomly-distributed BISO particles in the fuel compacts, an explicit stochastic geometry model was applied. Serpent reads the coordinates of the particles from a separate input file so that the particles are taken into account without approximations. The VHTRC specifications include a small gap of less than $1 \mathrm{~mm}$ between the fuel/graphite rods (i.e. the graphite sheath) and the surrounding graphite block. To simplify the model, this space was filled with block graphite. A cross section of a unit cell is shown in Figure 5.

Further assumptions made during the development of the Serpent model include the treatment of all carbon in all materials as graphite, i.e. the thermal scattering data for graphite is applied in all materials containing carbon. The thermal scattering data for uranium dioxide and the Dopplerbroadening rejection correction (DBRC) [10] for ${ }^{238} \mathrm{U}$ are also applied.

\subsection{Serpent model with a particle lattice}

In addition to the model with randomly distributed particles, a second Serpent model including a regular square particle lattice was developed (Figure 6). In an earlier study of the VHTRC, the particles in a square lattice were arranged in such a way that no particle was cut by the inner or outer fuel rod surface. In this model the packing fraction was retained on average in a fuel rod; however, the local packing fraction was slightly increased and some spots with only graphite emerged at the fuel rod inner and outer boundaries. As a result, differences of a few hundred $\mathrm{pcm}$ of the calculated eigenvalue in a comparison to the previously described random model appeared [11]. Therefore another particle lattice was created. Since this square lattice is infinitely filled with particles, some particles are clipped by the inner and the enclosing cylinder surfaces. The theoretical lattice pitch was determined from the theoretical packing fraction. In order to reduce the deviation from the correct amount of fuel in one fuel rod, this lattice pitch was slightly increased by about $0.46 \%$ and $0.05 \%$ for the B2 and B4 lattice, respectively.

For both Serpent models, 500,000 neutrons per cycle were calculated for 300 active cycles. The initial 40 cycles were skipped and not considered in the evaluation of the results. A combination of $5 \mathrm{MPI}$ and $31 \mathrm{OMP}$ processes typically led to simulation times of approximately 4 hours.

\subsection{SCALE/KENO-VI models}

The SCALE/KENO-VI continuous-energy (CE) models are identical to the Serpent lattice model. For the KENO-VI multi-group (MG) calculations, the DOUBLEHET cell data was specified to account for the double heterogeneous structure of the BISO particles in the fuel compact during 
the self-shielding calculation. The SCALE simulations included 50,000 neutrons in 500 active cycles and 50 initially skipped cycles. On a single processor, the CE KENO-VI simulations took about 25 hours and the MG calculations less than 8 hours.

In order to discard a sufficient number of initial neutron cycles in the Monte Carlo simulations, convergence of the multiplication factor and the Shannon entropy of the fission source distribution [12] were evaluated and ensured for all calculations.

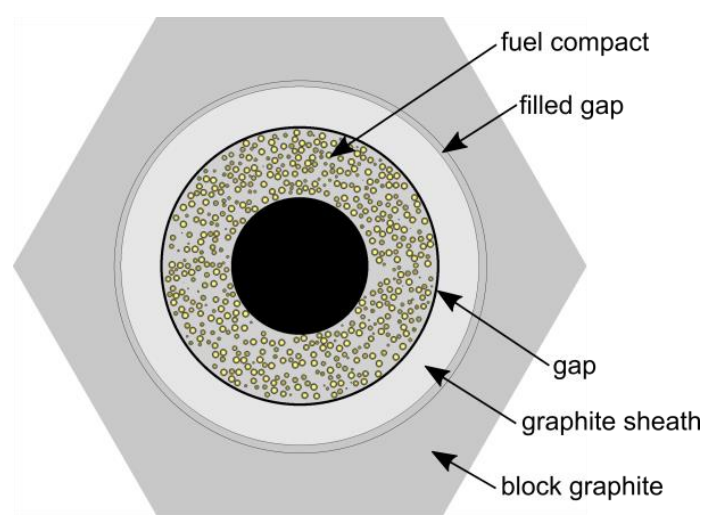

Figure 5: Cross sectional view of a B2 fuel unit cell with randomly distributed particles.

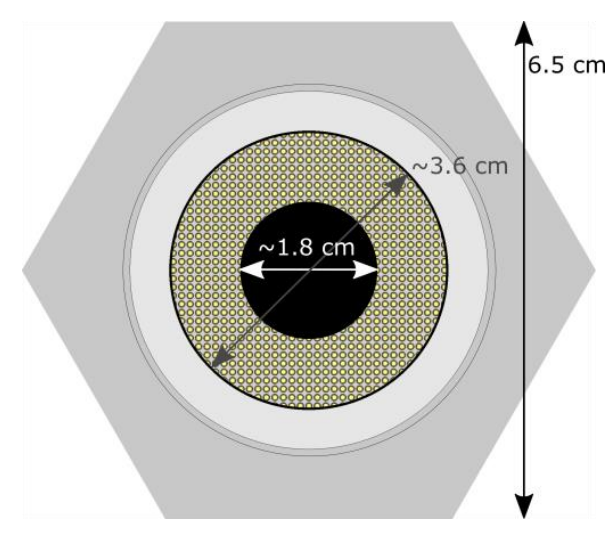

Figure 6: Cross sectional view of a B2 fuel unit cell including an infinite particle lattice with some particles being intersected by the inner and the enclosing cylinder.

\section{Results}

The multiplication factors of all calculated cores and states are compared as a function of temperature. All presented figures are subdivided into three sections to present the three cores next to each other. The experimental values are shown in all plots, and the results of the calculations with the Japanese continuous energy Monte Carlo code MVP-II, as reported in the VHTRC specifications [4], are also displayed when compared to our results.

\subsection{Serpent results}

\subsubsection{Calculations with ENDF/B-VII libraries}

With only one exception, the Serpent calculations show agreement within the $2 \sigma$ error bars between the random and the regular particle distributions for both libraries (Figure 7). Only the multiplication factors of the HC-2 core when using ENDF/B-VII.1 cross-sections show slightly increased differences, but still less than $50 \mathrm{pcm}$. The HC-2 core contains more fuel than the other cores, and the influence of the fuel particle distribution, i.e. the potential error in this modeling approach, might therefore be increased. It shall be mentioned that the influence of the geometric representation of HTGR particles within a graphite matrix has already been studied by, for example, F. Brown et al. [13]. In full core HTGR calculations, they found a difference of about $0.2 \%$ in the eigenvalue between models with infinite particle lattices (including intersected particles) and a MCNP5 stochastic geometry model. In contrast, the VHTRC calculations showed differences below $0.5 \%$ in all cores and for all considered temperatures. 
The calculations with the ENDF/B-VII.0 cross-sections show differences of up to $1,000 \mathrm{pcm}$ compared to the experiment. The corresponding ENDF/B-VII.1 calculations show significantly closer results to the experiment. Goto [14] has previously attributed eigenvalue differences of up to $0.9 \%$ in Monte Carlo simulations to an increase in the thermal neutron capture cross section of carbon in the JENDL-4.0 library compared to the earlier JENDL-3.3 release (see Table 1). This difference is even larger between the applied ENDF libraries than between the JENDL libraries. At a thermal energy of $0.025 \mathrm{eV}$, the capture cross section of carbon differs, for example, by about $0.5 \mathrm{mb}$ which leads to a significant decrease of the multiplication factor in the ENDF/B-VII.1 calculations. This is also confirmed when comparing the total capture reaction rate of carbon in the HC-2 core in terms of the library (Figure 8). Due to the increased capture cross section in the ENDF/B-VII.1 library, the capture reaction rate is increased at thermal energies.

Table 1: Neutron capture cross section of carbon at $0.0253 \mathrm{eV}$ [15].

\begin{tabular}{|l|l|l|l|l|l|}
\hline JEFF-3.1.2 & JEFF-3.2 & ENDF/B-VII.0 & ENDF/B-VII.1 & JENDL-3.3 & JENDL-4.0 \\
\hline $3.368 \mathrm{mb}$ & $3.816 \mathrm{mb}$ & $3.368 \mathrm{mb}$ & $3.861 \mathrm{mb}$ & $3.531 \mathrm{mb}$ & $3.861 \mathrm{mb}$ \\
\hline
\end{tabular}

In order to determine the main contributor to the decrease in the temperature-dependent eigenvalue for the HP core, the ratio of neutron capture in ${ }^{238} \mathrm{U}$, capture in graphite and leakage to the total neutron capture in the VHTRC was determined (see Table 2). The influence of the library on the neutron capture ratio of graphite is clearly visible. Afterwards the relative change of these values from the coldest to the hottest state, i.e. from $25.5^{\circ} \mathrm{C}$ to $199.6^{\circ} \mathrm{C}$, was calculated (see Table 3). Due to the Doppler broadening of the resonances, the neutron capture in ${ }^{238} \mathrm{U}$ is increased about 3.7\%. Conversely, the capture in graphite is significantly reduced since 1) there is increased competition from ${ }^{238} \mathrm{U}$ capture and 2) graphite capture is a typical 1/E absorber and experiences a neutron spectrum shift away from the 1/E region (i.e. as the core heats up, the thermal Maxwellian distribution and hence the average energy of thermal neutrons within the core increases). Apart from capture in the core, neutron leakage in this small geometry has significant influence: In comparison to the total neutron capture in the core, the number of neutrons that leave the VTHRC assembly increases by about $9 \%$ when the temperature is increased to $199.6^{\circ} \mathrm{C}$. Therefore, neutron leakage is found to be the main contributor to the eigenvalue decrease in the HP core.

Table 2: Ratio of neutron capture in ${ }^{238} \mathrm{U}$, neutron capture in graphite and neutron leakage to the total neutron capture in the VHTRC obtained with Serpent using the random particle distribution. The $1 \sigma$ standard deviations for all presented values are below $10^{-5}$.

\begin{tabular}{|l|l|l|l|l|}
\hline Library & Temperature & Ratio ${ }^{238} \mathrm{U} /$ total & $\begin{array}{l}\text { Ratio graphite/ } \\
\text { total }\end{array}$ & $\begin{array}{l}\text { Ratio Leakage/ } \\
\text { total }\end{array}$ \\
\hline \multirow{2}{*}{ ENDF/B-VII.0 } & $25.5^{\circ} \mathrm{C}$ & 0.315 & 0.276 & 0.293 \\
\cline { 2 - 5 } & $199.6^{\circ} \mathrm{C}$ & 0.327 & 0.255 & 0.320 \\
\hline \multirow{2}{*}{ ENDF/B-VII. 1} & $25.5^{\circ} \mathrm{C}$ & 0.317 & 0.313 & 0.288 \\
\cline { 2 - 5 } & $199.6^{\circ} \mathrm{C}$ & 0.329 & 0.289 & 0.315 \\
\hline
\end{tabular}


Table 3: Ratio of neutron capture in ${ }^{238} \mathrm{U}$, neutron capture in graphite and neutron leakage to the total neutron capture in the VHTRC obtained with Serpent using the random particle distribution: Relative change from the coldest to the hottest state. The $1 \sigma$ standard deviations for all presented values are below $0.002 \%$.

\begin{tabular}{|l|l|l|l|}
\hline Library & Capture in ${ }^{238} \mathrm{U}$ & Capture in graphite & Leakage \\
\hline ENDF/B-VII.0 & $3.72 \%$ & $-7.70 \%$ & $8.97 \%$ \\
\hline ENDF/B-VII.1 & $3.76 \%$ & $-7.59 \%$ & $9.31 \%$ \\
\hline
\end{tabular}

\subsubsection{Calculations with JEFF libraries}

Since the impact of the ENDF library version to the eigenvalues is large, there is an interest to evaluate the influence of the different cross section libraries. Therefore, additional calculations with the JEFF-3.1.2 and JEFF-3.2 cross section libraries were performed (Figure 9). The DBRC for ${ }^{238} \mathrm{U}$ and thermal scattering for $\mathrm{UO}_{2}$ could not be applied to these calculations. However, the influence of these treatments is small and therefore possible differences between JEFF and ENDF/B are only insignificantly influenced by this difference (see Table 4).

Table 4: Influence of the model or cross section library to the multiplications factor. All results refer to Serpent calculations using a random particle distribution and ENDF/B-VII.1 cross-sections, if not stated differently.

\begin{tabular}{|l|l|l|}
\hline & $\Delta\left(\mathrm{HP}, 199.6^{\circ} \mathrm{C}\right)[\mathrm{pcm}]$ & $\Delta\left(\mathrm{HC}-2,200.3^{\circ} \mathrm{C}\right)[\mathrm{pcm}]$ \\
\hline Particle lattice vs. random distribution & $18 \pm 11$ & $46 \pm 11$ \\
\hline Worth of 1mm gap & $195 \pm 11$ & $192 \pm 11$ \\
\hline MVP-II material vs. benchmark material & $60 \pm 11$ & $50 \pm 11$ \\
\hline Effect of DBRC & $18 \pm 11$ & $13 \pm 11$ \\
\hline Effect of thermal scattering in $\mathrm{UO}_{2}$ & $2 \pm 11$ & $29 \pm 11$ \\
\hline ENDF/B-VII.0 vs. ENDF/B-VII.1 & $1083 \pm 11$ & $964 \pm 11$ \\
\hline JEFF 3.1.2 vs. JEFF 3.2 & $979 \pm 11$ & $883 \pm 11$ \\
\hline
\end{tabular}

The ENDF/B-VII.0 and JEFF-3.1.2 libraries include identical neutron capture cross sections for carbon. Calculations with these libraries therefore produced results with a difference of less than $90 \mathrm{pcm}$ which is the result of slight differences in other cross sections. Furthermore, the neutron capture cross sections of carbon are similar in the ENDF/B-VII.1 and JEFF-3.2 library (difference less than $0.05 \mathrm{mb}$ ). Calculations with JEFF-3.2 cross sections therefore showed a similar trend compared to the two ENDF calculations: The eigenvalue is reduced by up to $1,000 \mathrm{pcm}$ compared to JEFF-3.1.2 calculations. The differences between the JEFF-3.2 and ENDF/B-VII.1 calculations are within $180 \mathrm{pcm}$. These calculations confirmed the observed eigenvalue trend in ENDF/B-VII (decrease up to 1,000 pcm), which depends on the library release and, thereby, the capture cross section of carbon. 


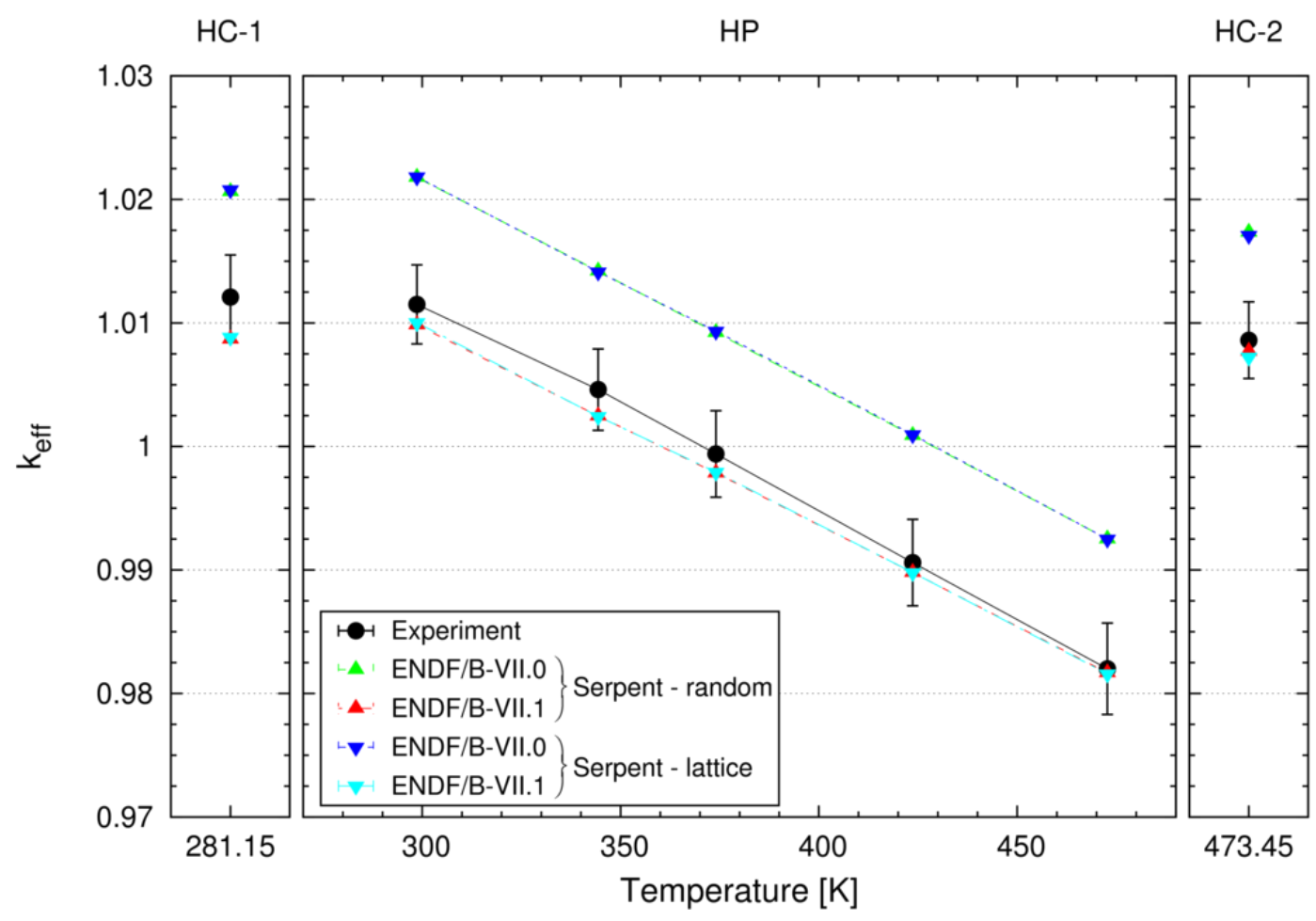

Figure 7: Comparison of Serpent and experimental VHTRC multiplication factors with $1 \sigma$ error bars.

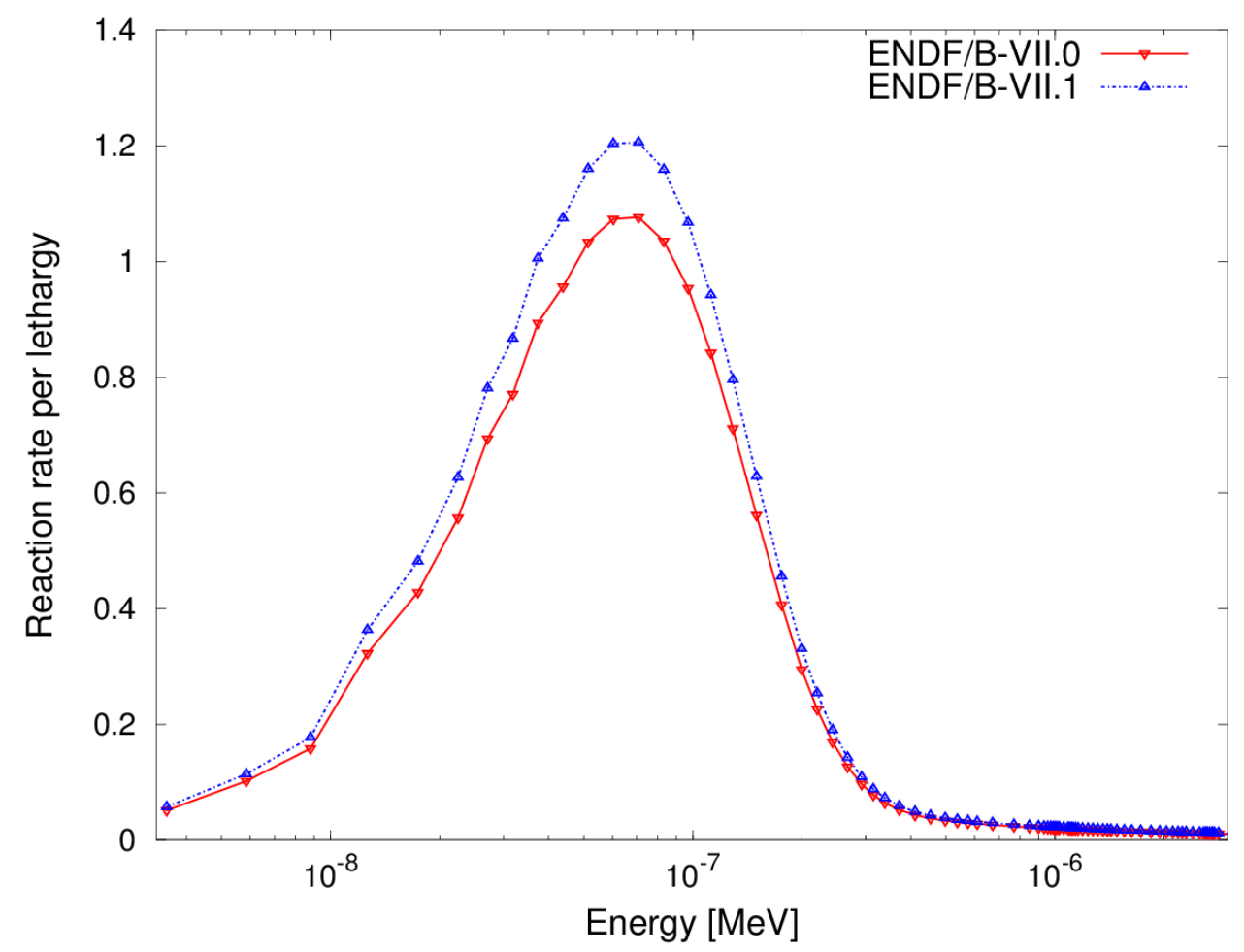

Figure 8: Capture reaction rate of carbon in the HC-2 core. 


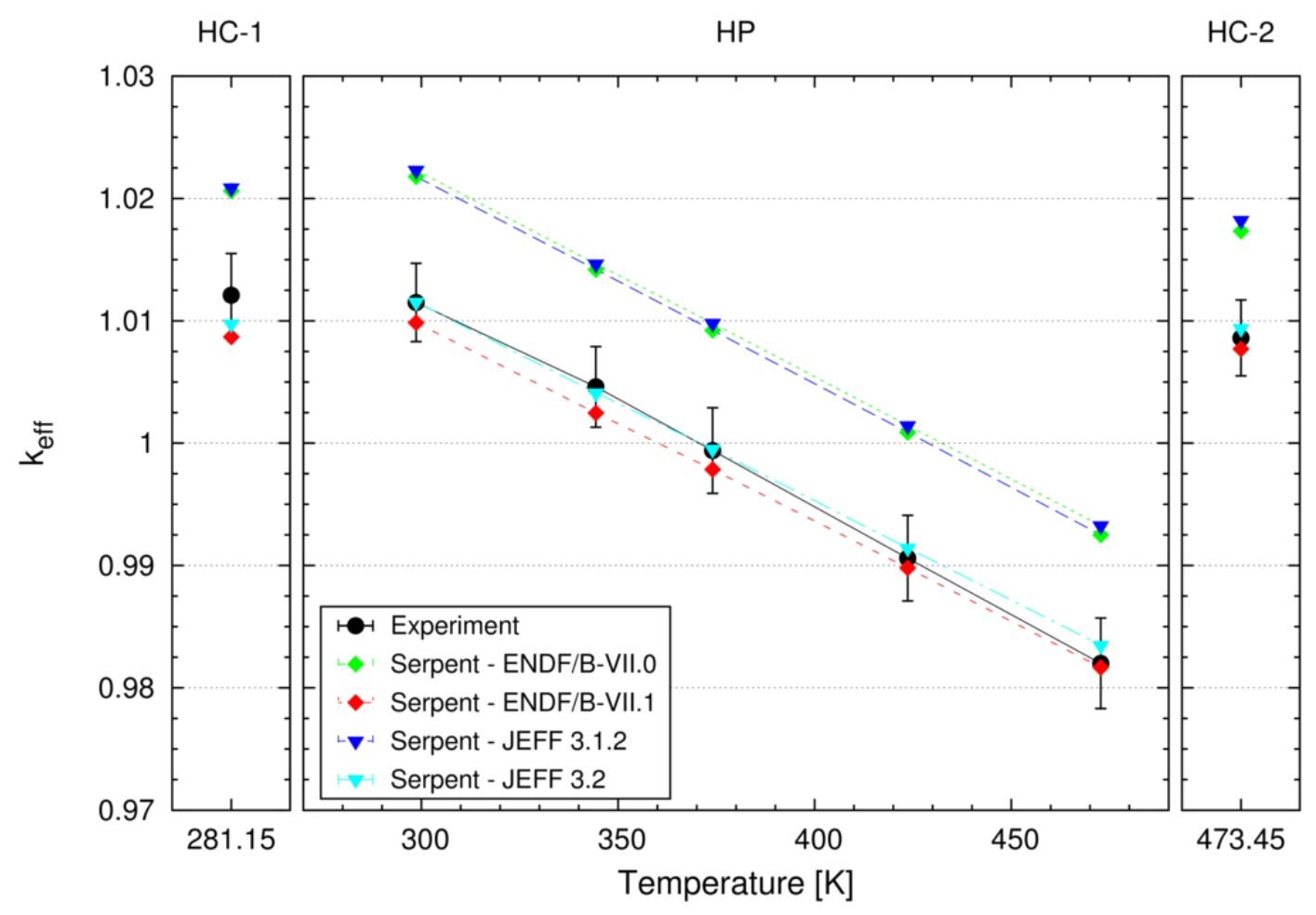

Figure 9: Comparison of Serpent 2 results with ENDF and JEFF cross-section data (random particle distribution).

\subsection{SCALE/KENO-VI CE results}

SCALE 6.1.2 is not capable of adjusting cross-sections as a function of temperature in CE calculations, and the code therefore uses cross section data from the closest available temperature point in the library. Since temperature interpolation becomes important when looking at small temperature differences in the HP experiments, comparisons with the CE calculations of SCALE 6.1.2 are therefore not included.

In SCALE 6.2b4 the problem-dependent Doppler-broadening option was applied [16], and the corresponding CE calculations therefore show a continuous decrease of the eigenvalue with increasing temperature (Figure 10). The slight unevenness in the decrease can mainly be attributed to the temperature interpolation scheme of the $S(\alpha, \beta)$ values of the moderating graphite [17]. This was confirmed by an additional set of calculations using a hypothetical model that replaces graphite by free-gas carbon: for these cases, it was found that the decrease of $k_{\text {eff }}$ with the temperature is linear. It shall, however, be emphasized that these calculations were only performed to track down the reason for the non-linearity in the $k_{\text {eff }}$ curve; the results based on the free-gas carbon assumption are clearly unphysical and therefore not included here.

A comparison between SCALE 6.2b4 results to the corresponding Serpent model revealed differences of $300-550 \mathrm{pcm}$ for both libraries. These differences are suggested to be the results of the previously mentioned temperature interpolation of graphite cross sections, and the 
application of cross section libraries that are prepared via different nuclear data processing systems.

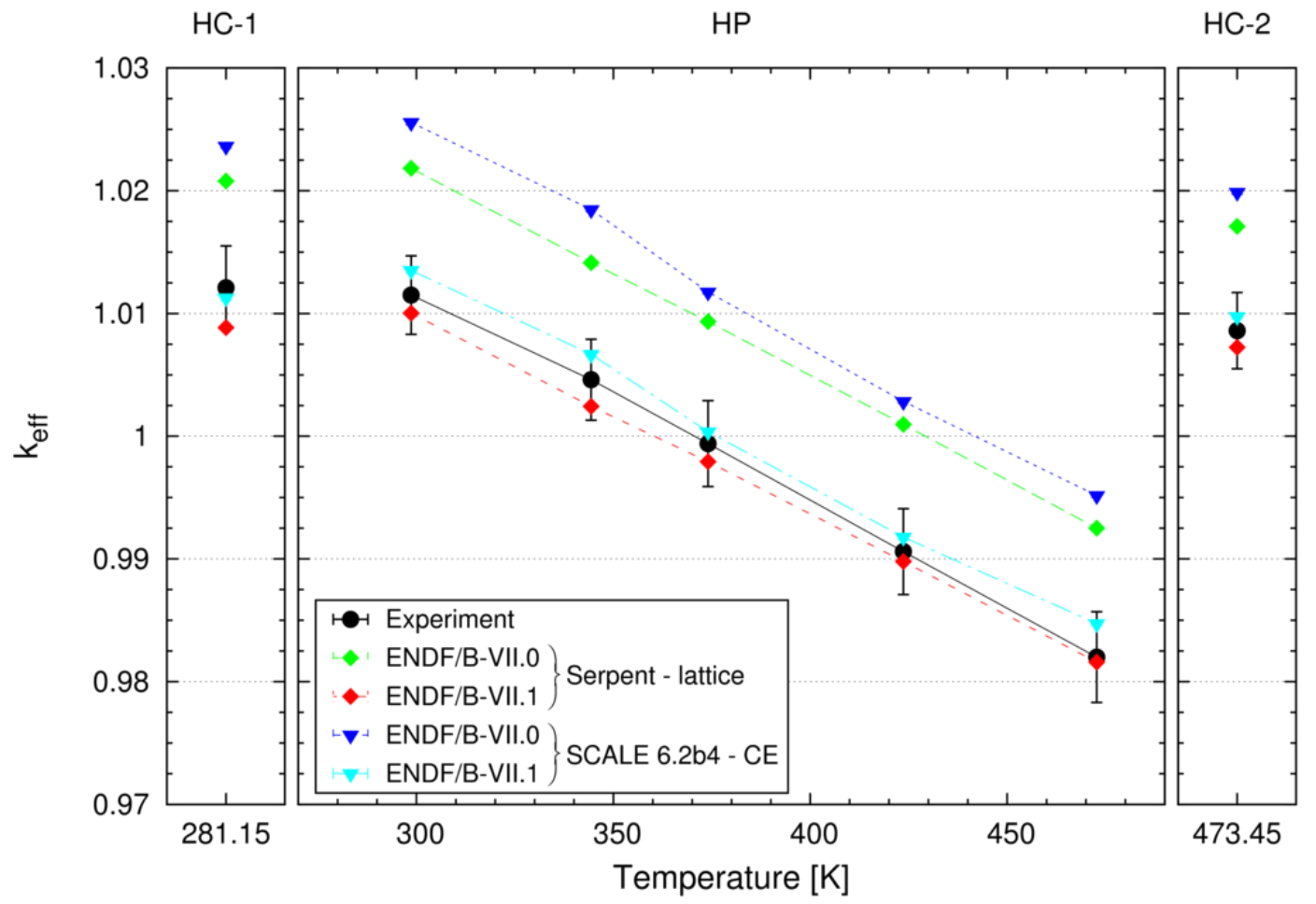

Figure 10: Comparison of SCALE/KENO-VI CE and the corresponding Serpent VHTRC multiplication factors.

\subsection{SCALE/KENO-VI MG results}

The standard DOUBLEHET treatment in SCALE/KENO-VI does not allow for the direct treatment of annular double heterogeneous fuel compacts. However, the control sequence for criticality calculations with KENO automatically generates inputs for the modules that calculate the self-shielded cross sections. These inputs were modified (without changing the source code) to take the central hole in the fuel rod into account in the self-shielding calculation.

The differences between the 238-group SCALE 6.1.2 calculations and the Serpent random calculations are less than $150 \mathrm{pcm}$ when using the ENDF/B-VII.0 library, while the differences between the corresponding SCALE 6.2b4 calculations are less than $60 \mathrm{pcm}$ (Figure 11). The 252-group calculations show differences of less than $180 \mathrm{pcm}$ for both ENDF libraries (Figure 11 and Figure 12). 


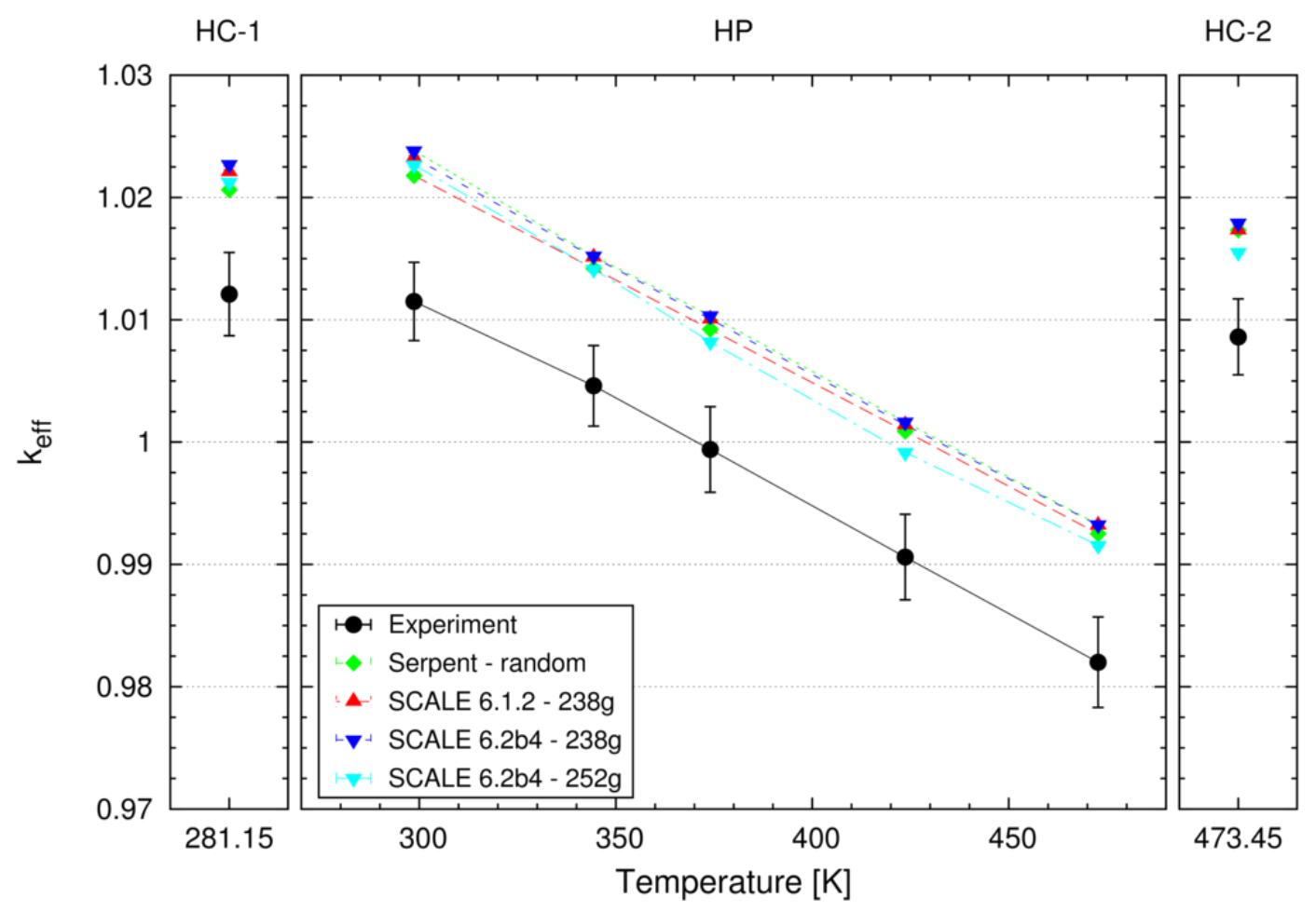

Figure 11: Comparison of the SCALE/KENO-VI MG and Serpent VHTRC multiplication factors with ENDF/BVII.0 cross-sections.

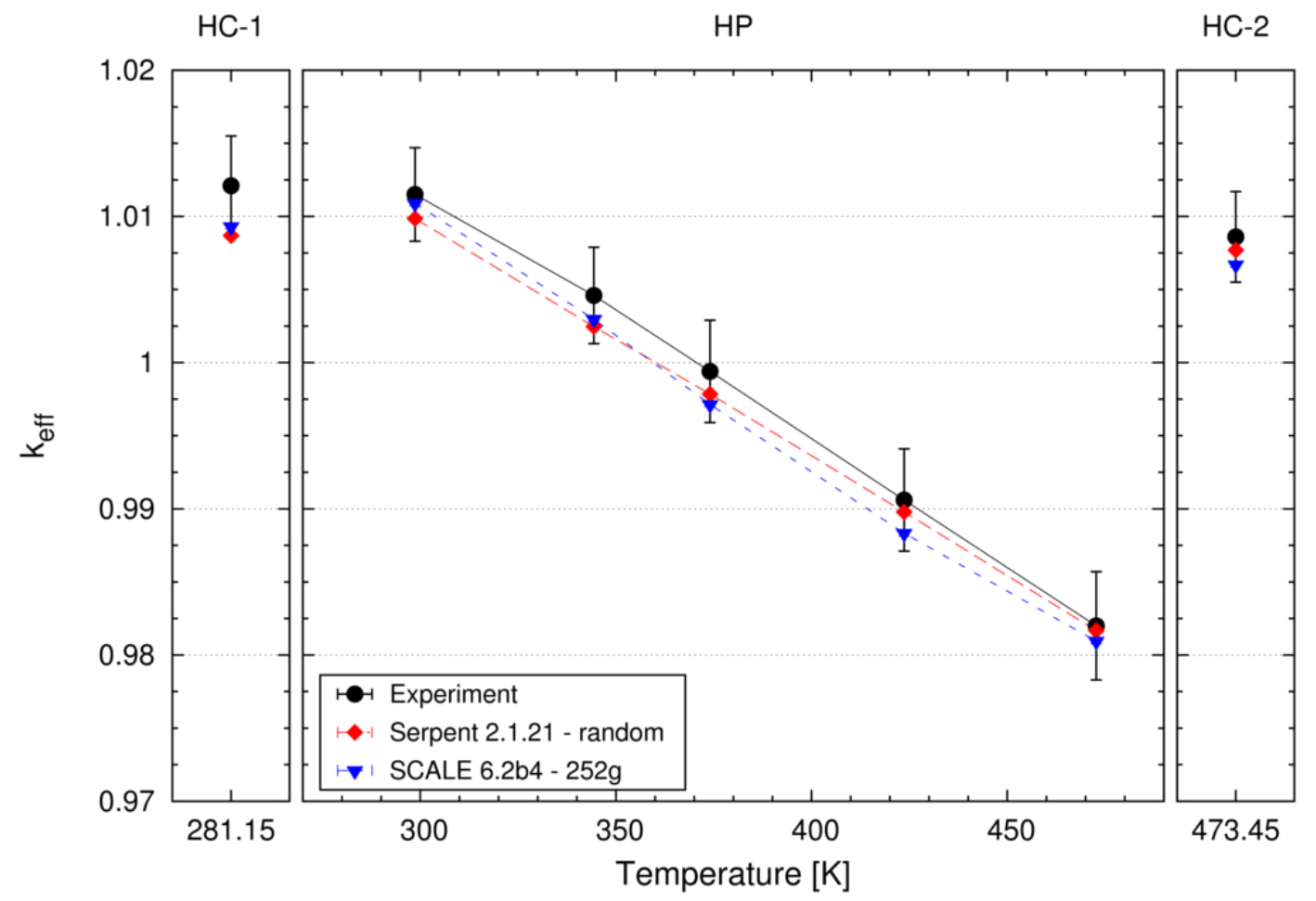

Figure 12: Comparison of the SCALE/KENO-VI 252-group and Serpent VHTRC multiplication factors with ENDF/B-VII.1 cross-sections. 


\subsection{Comparison to MVP-II calculations and the experiment}

A comparison with calculations of the Japanese Monte Carlo code MVP-II with JENDL-4.0 continuous-energy cross sections (as published in the IRPhEP specifications of the VHTRC) show a consistent underestimation of the experimental values by $300-500 \mathrm{pcm}$ for the HP and $\mathrm{HC}-2$ cores, and by about $700 \mathrm{pcm}$ for the HC-1 core. The MVP-II data sets are compared to the Serpent calculations using the ENDF/B-VII.1 data library and random particle distributions (Figure 13) because the neutron absorption cross section of graphite has also been corrected in the JENDL-4.0 library [14] and the MVP-II model utilized a random particle distribution. As mentioned earlier, the model that was initially created for the IAEA CRP (referred to here as CRP model) filled the $1 \mathrm{~mm}$ gaps between the fuel rods and the graphite block with graphite. The amount of graphite is therefore slightly increased and moderation improved. A better comparison to the MVP-II calculation is achieved with a model that includes these gaps. This modification leads to multiplication factors about $200 \mathrm{pcm}$ smaller and therefore closer to the MVP-II model.

It was further noticed that the material specifications, as indicated in the published MVP-II input deck, are slightly different from the final benchmark specifications, and a third set of calculations was therefore performed with the MVP-II material data. The change of the material definition led to decrease of the eigenvalues between $30-60 \mathrm{pcm}$. The resulting difference to the MVP-II calculation decreased to less than $50 \mathrm{pcm}$ for the HP core, and about $100 \mathrm{pcm}$ for the other cores.

The closest Serpent model to the benchmark specifications would utilize a random particle distribution, the latest release of the ENDF-B-VII cross-section library and include the gaps. This model shows differences of a few hundred pcm to the experimental data set (see Figure 13). Some multiplication factors are within the $1 \sigma$ error bars of the experiment $(1 \sigma$ is about $350 \mathrm{pcm}$ ) while the others are only slightly outside them. Therefore the obtained results are considered to be in good agreement with the experiment. Remaining differences might be caused by the impact of the nuclear data library and the applied cross-section generation method, as already discussed earlier. The differences of the multiplication factors obtained by Serpent and MVP-II to the benchmark values are additionally presented in Figure 14. 


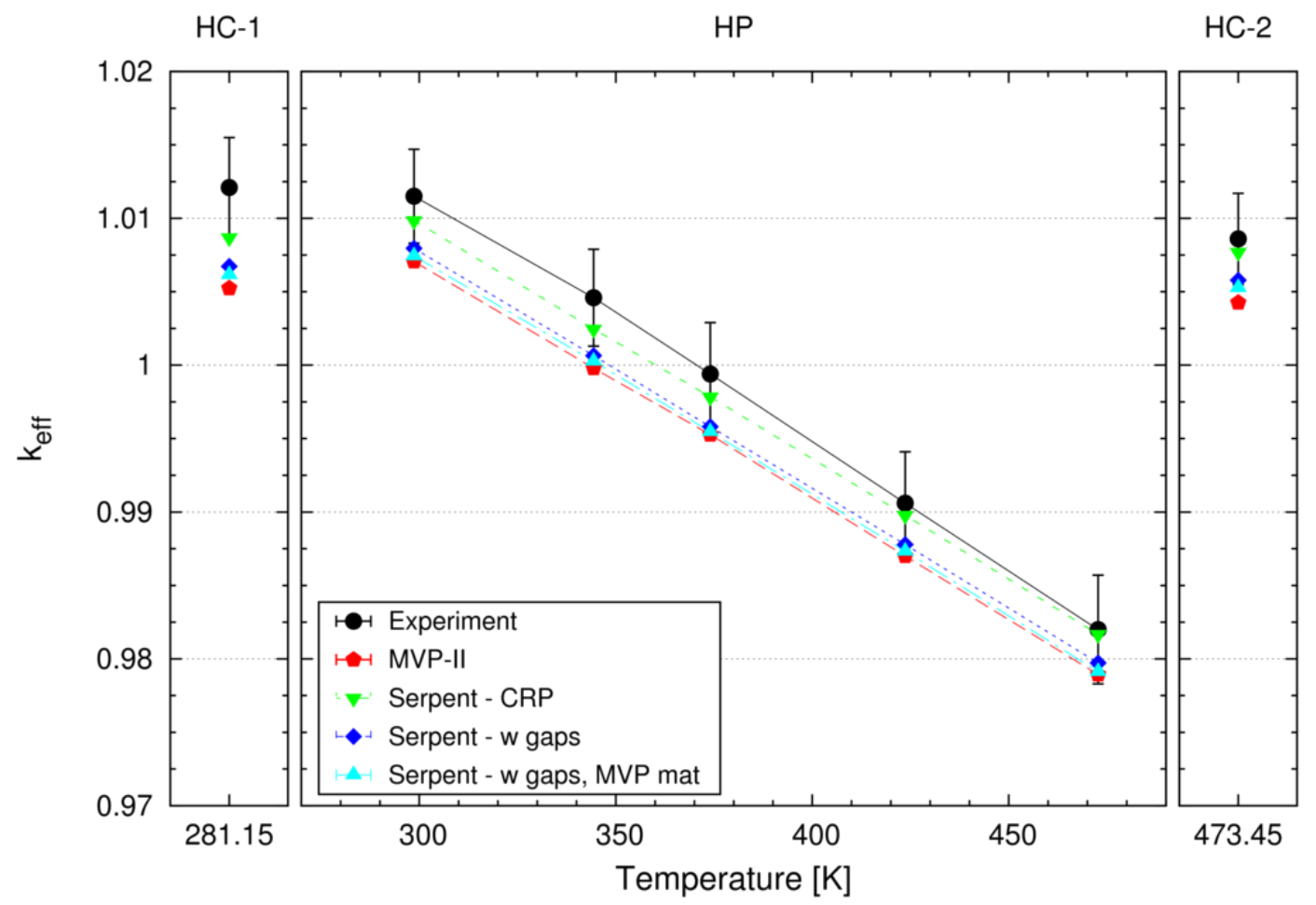

Figure 13: Comparison of the Serpent (ENDF/B-VII.1) and MVP-II (JENDL-4.0) [4] VHTRC multiplication factors.

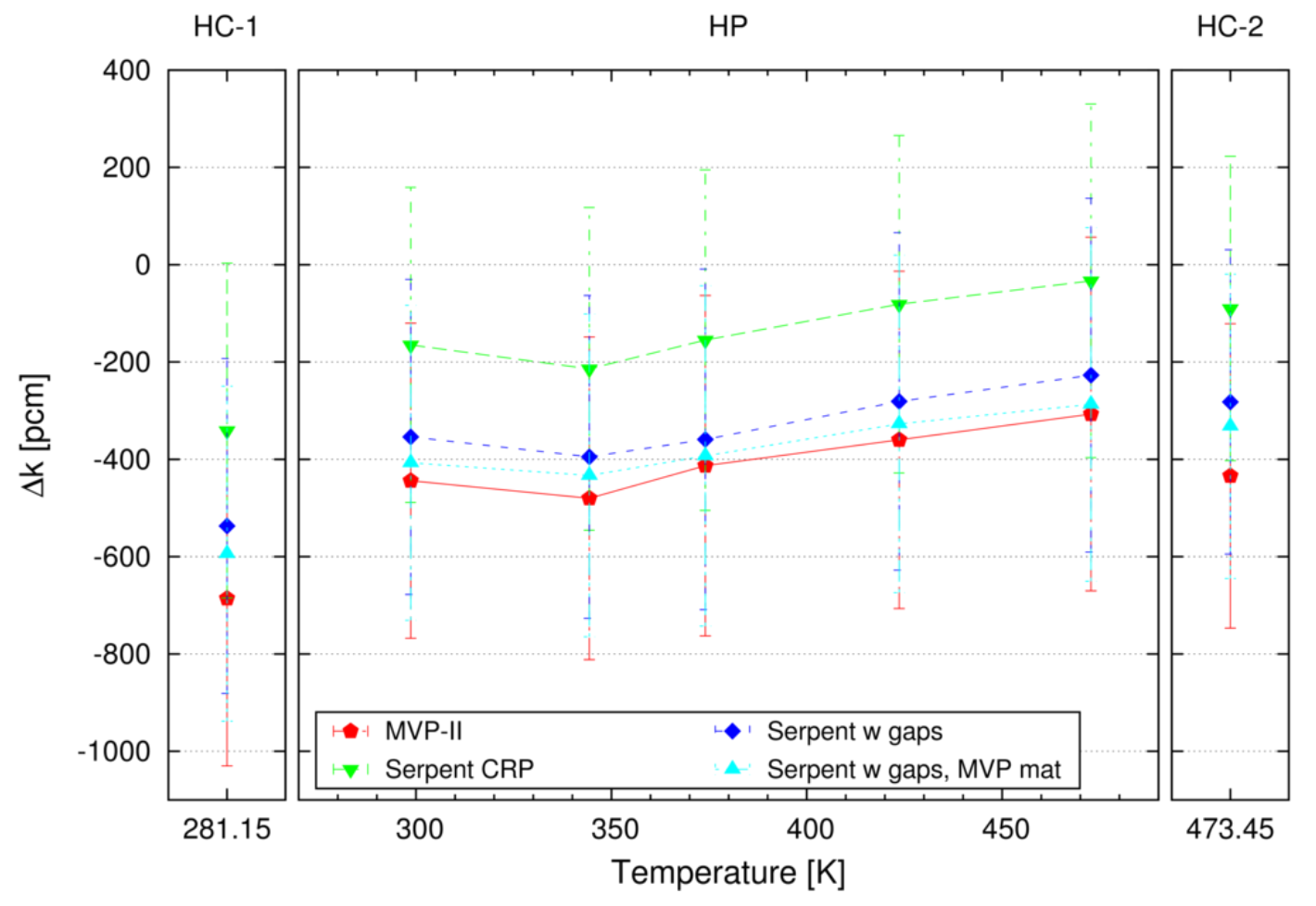

Figure 14: Difference of the multiplication factors of Serpent (ENDF/B-VII.1) and MVP-II (JENDL-4.0) to the experimental multiplication factors. 


\subsection{Fission reaction rate}

In addition to the eigenvalue, the axial and radial fission reaction rates were investigated in order to provide an additional quantity for comparison between the codes and models. For this study, four fuel rods of the $\mathrm{HC}-2$ core were investigated as indicated in Figure 15.

The Serpent and KENO-VI results of the axial fission rate distributions in the innermost of the rods show agreement within $2 \sigma$ error bars. The same is valid for the radial fission rate distribution. Due to this agreement and limited space, only the distributions obtained with Serpent using the random particle distribution and ENDF/B-VII.1 cross section are included in Figure 16 and Figure 17.

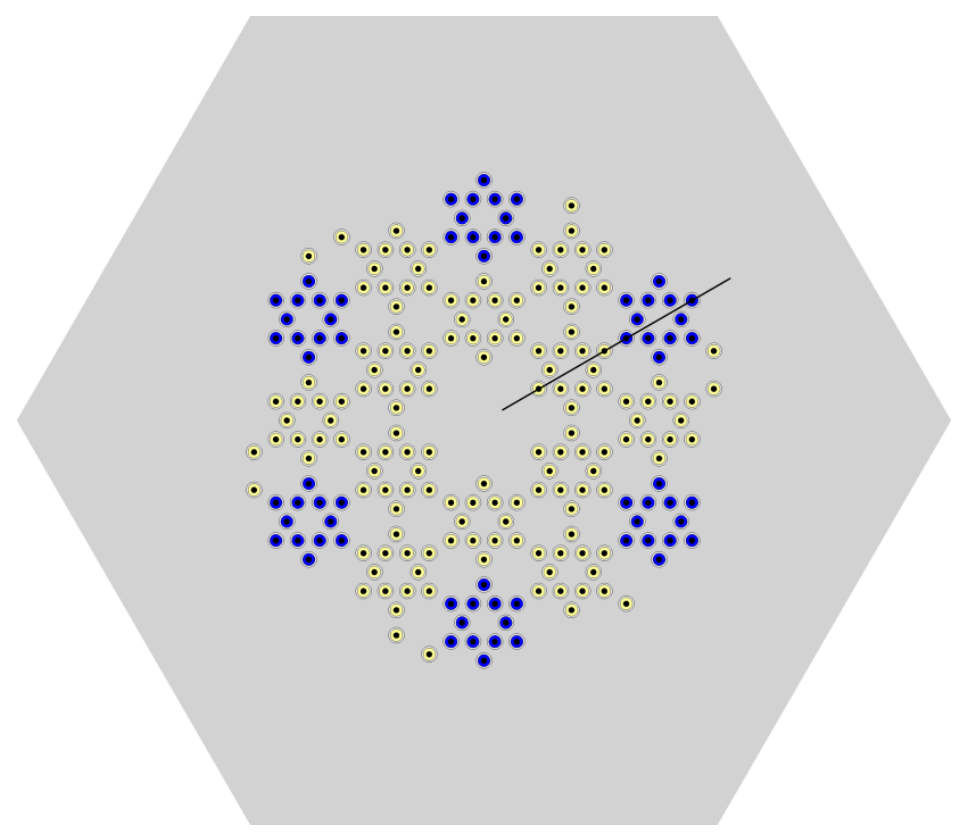

Figure 15: HC-2 core: Yellow (light) and blue (dark) fuel rods indicate B4 and B2 fuel, respectively. The line indicates the investigated fuel rods. 


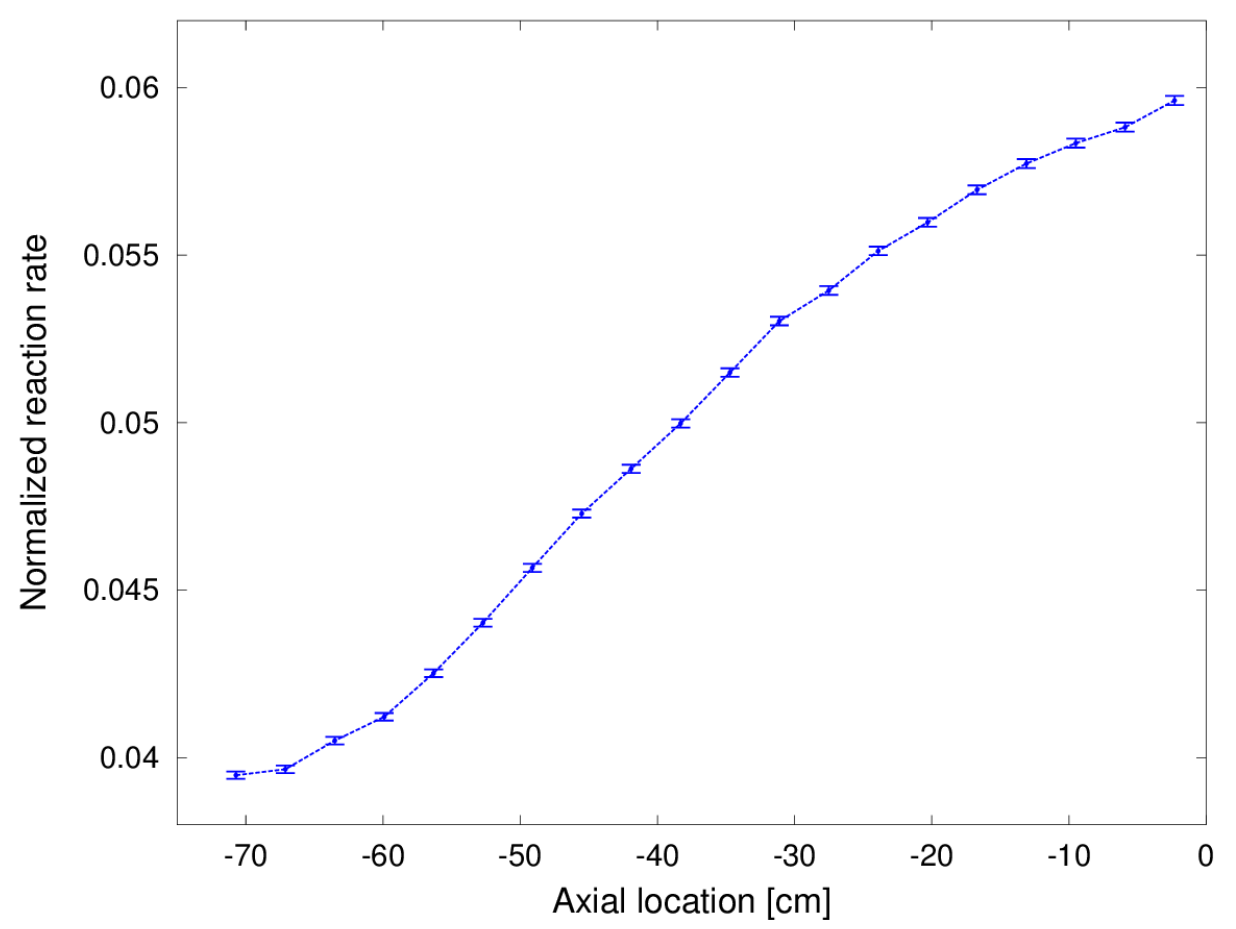

Figure 16: Axial distribution of the fission reaction rate in the innermost of the investigated HC-2 rods obtained with Serpent. The abscissa starts at the bottom of the fuel rod in the movable half of the VHTRC core; $\mathbf{0 ~} \mathrm{cm}$ indicates the interface between the half blocks.

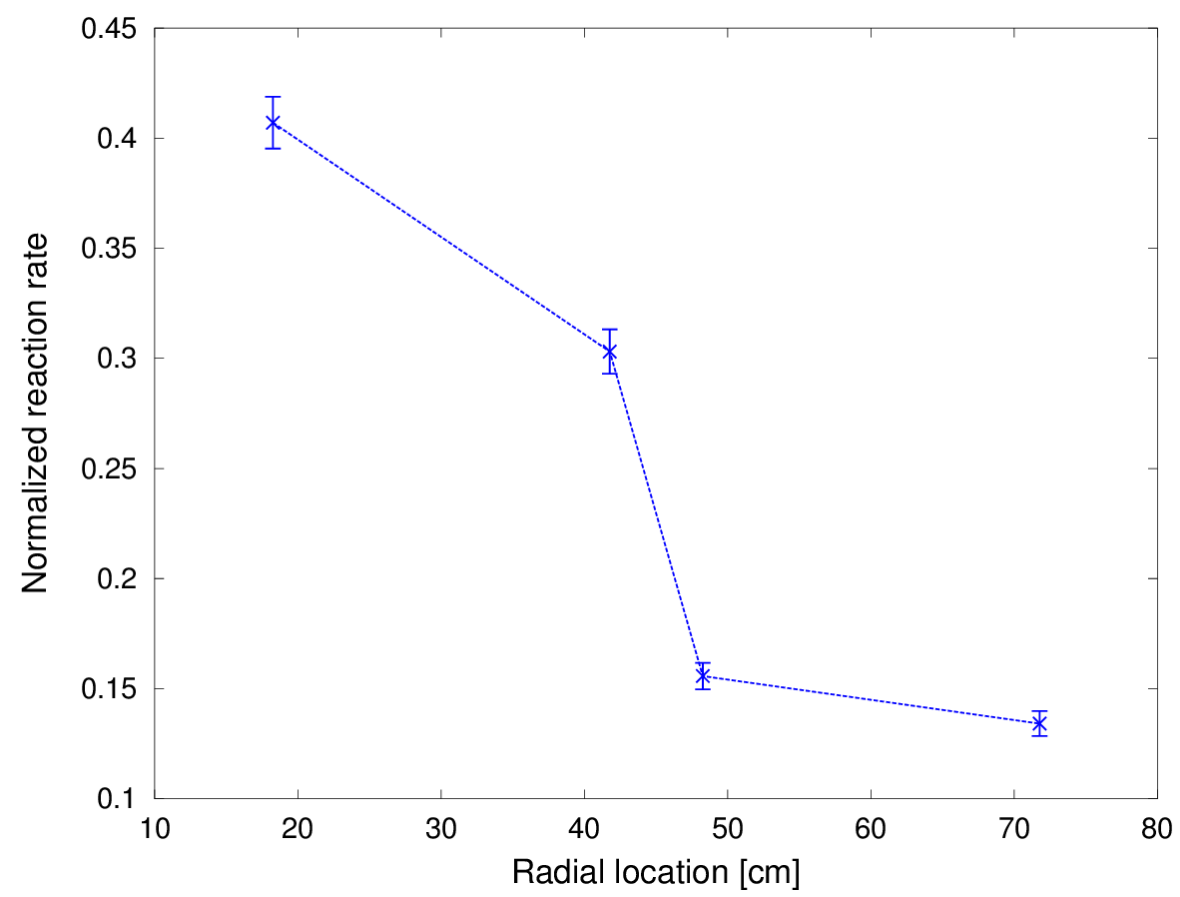

Figure 17: Radial distribution of the fission reaction rate in the HC-2 core obtained with Serpent. The abscissa starts at the inner section of the core and moves outwards; $0 \mathrm{~cm}$ would indicate the radial center of the core (not displayed). 


\subsection{Doppler coefficient}

In addition to the eigenvalue, the benchmark data set includes the Doppler coefficients of reactivity. The Doppler coefficient between the coldest and the hottest HP state, i.e. between $25.5^{\circ} \mathrm{C}$ and $199.6^{\circ} \mathrm{C}$, was compared with the Serpent and KENO-VI calculations. The formula that was applied for the determination of this coefficient is the following:

$$
\alpha_{T}=\frac{\frac{1}{k_{199.6}}-\frac{1}{k_{25.5}}}{\Delta T}
$$

with the eigenvalues $k$ at the respective temperature points and $\triangle T=174.1 \mathrm{~K}$. It was found that the Serpent, KENO-VI CE and KENO-VI 238-group calculation with SCALE 6.1.2 underestimate the experimental Doppler coefficient by less than $5 \%$. In contrast, the 238- and 252-MG calculations with SCALE 6.2b4 overestimate the experimental value by $1-3 \%$. All Doppler coefficient calculations therefore show agreement with the experiment within the $2 \sigma$ standard deviations.

Table 5: Doppler coefficient between $25.5^{\circ} \mathrm{C}$ and $199.6^{\circ} \mathrm{C}$ in the HP core.

\begin{tabular}{|c|c|c|c|}
\hline & & $\alpha_{\mathrm{T}} \pm 1 \sigma[\mathrm{pcm} / \mathrm{K}]$ & $(\mathrm{C}-\mathrm{E}) / \mathrm{E}$ \\
\hline Experi & & $17.10 \pm 0.6$ & (ref) \\
\hline MVP-I & & $16.39 \pm 0.05$ & $-4.16 \%$ \\
\hline & Serpent random & $16.58 \pm 0.06$ & $-3.05 \%$ \\
\hline & SCALE/KENO-VI CE 6.2b4 & $16.88 \pm 0.14$ & $-1.26 \%$ \\
\hline$\stackrel{m}{1}$ & SCALE/KENO-VI 238g 6.1.2 & $16.97 \pm 0.14$ & $-0.75 \%$ \\
\hline$\overline{\mathrm{Q}} \stackrel{\circ}{=}$ & SCALE/KENO-VI 238g 6.2b4 & $17.27 \pm 0.12$ & $0.98 \%$ \\
\hline ш $>$ & SCALE/KENO-VI 252g 62b4 & $17.62 \pm 0.14$ & $3.02 \%$ \\
\hline & Serpent random & $16.33 \pm 0.06$ & $-4.53 \%$ \\
\hline$\stackrel{m}{u}$ & Serpent lattice & $16.42 \pm 0.07$ & $-3.95 \%$ \\
\hline $\bar{\eta}$ & SCALE/KENO-VI CE 6.2b4 & $16.60 \pm 0.15$ & $-2.91 \%$ \\
\hline $\bar{\Psi}$ & SCALE/KENO-VI 252g 62b4 & $17.35 \pm 0.15$ & $1.45 \%$ \\
\hline
\end{tabular}

\section{Summary and Conclusion}

For the validation of the cell and lattice calculations of Exercises I-1 and I-2 in Phase I of the IAEA CRP on HTGR UAM, criticality simulations of the VHTRC were performed with the Monte Carlo codes Serpent 2 and SCALE/KENO-VI.

Due to the application of problem-dependent temperature correction in SCALE/KENO-VI 6.2b4 continuous-energy calculations, reasonable agreement was observed in comparisons with the corresponding Serpent calculations. Differences between 300 and $550 \mathrm{pcm}$ were obtained.

The SCALE 6.2b4 calculations with the new 252-group library show differences of less than 180 pcm for both ENDF libraries. Slightly larger differences were obtained with the 238-group library. SCALE 6.1.2 produced differences less than $150 \mathrm{pcm}$ in corresponding calculations. It should, however, be kept in mind that all SCALE 6.2 calculations were performed using a beta version of 
the code. This code and the data might still change until its final release, and the results obtained should be regarded as preliminary.

The Japanese MVP-II Monte Carlo calculations with JENDL-4.0 CE data revealed a consistent underestimation of the experiment and all simulations performed in this work. The addition of the gaps between the fuel and graphite rods and the surrounding graphite block in the model, and the use of the MVP-II material compositions with ENDF/B-VII.1 cross sections led to good agreement between the MVP-II and the Serpent random calculations.

All simulations utilizing the ENDF/B-VII.0 cross-sections are about $1 \%$ higher than the experimental data. The Monte Carlo calculations using the ENDF/B-VII.1 library produced significantly closer results to experimental values. The increase of the neutron capture cross section in graphite in this library version compared to its prior release has a crucial impact on the calculations of the multiplication factor of this graphite moderated assembly.

For reasons of simplification, the VHTRC model that was initially developed for the IAEA CRP does not consider gaps between graphite/fuel rods and the surrounding block graphite. The addition of graphite in these gaps lead to an eigenvalue increase of about $200 \mathrm{pcm}$ compared to a model with gaps. The investigated fission reaction rate distributions are consistent regarding the $2 \sigma$ standard deviation for all investigated models and codes, and the Doppler coefficients also match the experimental values within $2 \sigma$ standard deviations. The SCALE/KENO-VI inputs generated for this study shall serve as the basis for e.g. the SCALE/TSUNAMI inputs for uncertainty calculations at a later stage, and the impact of this simplification should therefore also be examined during these studies.

\section{Acknowledgements}

This work was supported by the German Federal Ministry for Economic Affairs and Energy, and the U.S. Department of Energy, Assistant Secretary for the Office of Nuclear Energy, under DOE Idaho Operations Office Contract DE-AC07-05ID14517.

\section{References}

[1] F. Reitsma, et al., "The IAEA Coordinated Research Program on HTGR Uncertainty Analysis: Phase I Status and Initial Results", Proceedings of HTR 2014, Weihai, China, 2014.

[2] K. Ivanov, et al., "Benchmark for Uncertainty Analysis in Modeling (UAM) For Design, Operation and Safety Analysis of LWRs. Volume I: Specification and Support Data for the Neutronics Cases (Phase I)", OECD Nuclear Energy Agency, NEA/NSC/DOC(2013)7, May 2013.

[3] J. Ortensi, et al., "Prismatic Coupled Neutronics/Thermal Fluids Transient Benchmark of the MHTGR-350 MW Core Design: Benchmark Definition”, NEA/NSC/DOC, 2013. 
[4] NEA, "International Handbook of Evaluated Reactor Physics Benchmark Experiments", NEA/NSC/DOC, NEA No. 7140, 2013.

[5] J. Leppänen, "Development of a New Monte Carlo Reactor Physics Code", PhD Thesis, Helsinki University of Technology, 2007.

[6] "SCALE: A comprehensive modeling and simulation suite for nuclear safety analysis and design", ORNL/TM-2005/39, Version 6.1, Oak Ridge National Laboratory, Oak Ridge, Tennessee, USA, June 2011.

[7] F. Bostelmann, et al., "Sampling-based Nuclear Data Uncertainty Analysis in Criticality and Depletion Calculaitons", Proceedings of the ANS MC2015, Nashville, TN, USA, April 19-23, 2015.

[8] J. Leppänen, et al., "The Serpent Monte Carlo code: Status, development and applications in 2013", Annals of Nuclear Energy, Vol. 82, pp. 142-150, 2015.

[9] B. T. Rearden, et al., "Overview of SCALE 6.2", Proceedings of ANS NCSD 2013, Wilmington, NC, USA, September 29 - October 3, 2013.

[10] M. Ouisloumen, R. Sanchez, "A model for neutron scattering off heavy isotopes that accounts for thermal agitation effects", Nuclear Science and Engineering, Vol. 107, No. 3, pp. 189-200, 1991.

[11] G. Strydom, et al. „Results for Phase I of the IAEA Coordinated Research Program on HTGR Uncertainties", INL/EXT-14-32944, Revision 2, August 2015.

[12] F. B. Brown, "On the Use of Shannon Entropy of the Fission Distribution for Assessing Convergence of Monte Carlo Criticality Calculations", Proceedings of PHYSOR 2006, Vancouver, Canada, September 10-14, 2006.

[13] F. B. Brown, et al., "Stochastic Geometry and HTGR Modeling with MNCP5", Proceedings of The Monte Carlo Method, Chattanooga, TN, USA, April 17-21, 2005.

[14] M. Goto, et al., "Impact of revised thermal neutron capture cross section of carbon stored in JENDL-4.0 on HTTR criticality calculation", Journal of Nuclear Science and Technology, Vol. 48, No. 7, pp. 965-969, 2011.

[15] JANIS 4.0, http://www.oecd-nea.org/janis/, 2013

[16] B. T. Rearden, et al., "Enhancements in Continuous-Energy Monte Carlo Capabilities for SCALE 6.2", Proceedings of PHYSOR 2014, Kyoto, Japan, September 28 - October 3, 2014.

[17] S. W. D. Hart, "Automated Doppler Broadening of Cross Sections for Neutron Transport Applications", PhD thesis, University of Tennessee, 2014. 